\title{
A NEW ALGORITHM TO CONSTRUCT S-BOXES WITH HIGH DIFFUSION
}

\author{
Claudia Peerez Ruisanchez \\ Universidad Autonoma del Estado de Morelos
}

\begin{abstract}
In this paper is proposed a new algorithm to construct S-Boxes over GF(28) with Branch Number value at least 3. This is an important property that guarantees a high diusion in the S-Box [12]. Also are introduced some defnition and properties that show the be-havior of S-Boxes under the composition with affine functions. Finally is presented a comparision between this algorithm and the method pro-posed by Tavares [12].
\end{abstract}

\section{INTRODUCATION}

In his paper Communication Theory of Secrecy Systems", Claude Shannon defines confusion and diffusion as two essential properties for block cipher design. S-Boxes to be used in this cryptographic algorithms require the compliance of criteria such as Nonlinearity, Branch Number, Algebraic Degree and Differential Characteristic, which bring security against differential and linear cryptanalysis [9][10].

In 1994 Tavares [12] proposes a method to and S-Boxes with a desired value of Branch Number. The principal problem of this proposal is that the obtained S-Boxes can have a big number of fixed points and his Nonlinearity values were small to be used in Block Ciphers.

In this paper we propose a new algorithm to construct S-Boxes with Branch Number at least 3. In this method the obtained S-Boxes doesn't have fixed points and his Nonlinearity values are acceptable. Also we present the definition of Parameter Set and introduce some properties that allow show when the parameter set of a S-Box is invariant under the composition with affine transformations. This have an special importance because we can obtain dynamic S-Boxes for Block Ciphers constructing one with good Parameter Set and making the composition with a_ne functions.

\section{THEORETICAL ASPECTS}

In this section are presented some definitions related with S-boxes design that are very important for a better understanding of the paper. 
International Journal of Soft Computing, Mathematics and Control (IJSCMC),Vol. 4, No. 3, August 2015

Definition 1. A function II : $\mathrm{GF}(2)^{\mathrm{n}} \rightarrow \mathrm{GF}(2)^{\mathrm{m}}$ is called an $n \times m$ S-Box, in which any input of $n$ bits is transformed into output of $m$ bits

There are many criteria dedicated to measure the cryptographic security of S-Boxes. However, does not exists an universal measure that allow affirm which of this are the most important to take into account in S-Box design. We will work with the four criteria presented bellow [11]:

Definition 2 (Nonlinearity). Let $\mathrm{f}:(\mathrm{GF}(2))^{\mathrm{n}} \rightarrow(\mathrm{GF}(2))^{\mathrm{n}}$ the nonlinearity of this function denoted by $n l(f)$ is the magnitude:

$$
n l(f)=\min _{g \in A_{n}} w t(f+g)
$$

when $A_{n}$ is the set of affine functions and wt is the Hamming weight.

Definition 3 (Algebraic Degree). Let $\mathrm{f}\left(x_{1} ; x_{2} \ldots \ldots x_{\mathrm{n}}\right)$ a boolean function of $n$ variables, then $f$ could be represented as (ANF):

$$
f(x)=a_{0} \oplus \bigoplus_{i=1}^{n} a_{i} x_{i} \oplus \bigoplus_{1 \leq i \neq j \leq n} a_{i j} x_{i} x_{j} \oplus \ldots . \oplus a_{12 \ldots n} x_{1} x_{2} \ldots x_{n}
$$

when the coefficients $a 0, a \mathrm{i}, a \mathrm{ij}, \ldots \ldots ., a 12 \ldots . . n €\{0,1\}$. The number of variables in the product of higher order with non-zero coefficients is known as algebraic degree of $f$.

The algebraic degree of a vectorial boolean function is the minimum algebraic degree of all nonzero linear combinations of his component functions.

$$
\operatorname{deg}(S)=\min _{f} \operatorname{deg}\left\{f \mid f=\bigoplus_{i=1}^{m} j_{i} f_{i}(x)\right\}
$$

Definition 4 (Differential Characteristic). Let $G F(2)^{n}$ y $G F(2)^{m}$ two finite vectorial spaces. An application $\Pi: G F(2)^{n} \rightarrow G F(2)^{m}$ is called differential $\delta$ - uniform (or differential characteristic $\delta$ ) if $\forall \alpha \in G F(2)^{n}, \alpha \neq 0$ and $\beta \in G F(2)^{m},\left|\left\{z \in G F(2)^{n} \mid \Pi(z+\alpha)+\Pi(z)=\beta\right\}\right| \leq \delta$.

Definition 5 (Branch Number). The branch number (diffusion coefficient) of $f: G F(2)^{n} \rightarrow G F(2)^{n}$ is defined as the value obtained from the expression:

This four criteria: branch number, algebraic degree, nonlinearity and differential characteristic are very important because they provide the necessary elements against the linear and differential cryptanalysis. 
International Journal of Soft Computing, Mathematics and Control (IJSCMC),Vol. 4, No. 3, August 2015

Linear Cryptanalysis The main objective of linear cryptanalysis if to find a linear probabilistic relation for a part of the cipher equation that connects the input and the output data. Also this relation must be complied with a probability significantly different of $1 / 2$.

If this probability is denoted by $p L$ is necessary minimize the magnitude $\mid p_{L}-1 / 2 \|$ to obtain a good security against this kind of attack. If we assume that the $\mathrm{X}$ was selected randomly, then; the better linear approximation of an S-Box in the form $a_{1} X_{n} \oplus \ldots \oplus a_{n} X_{n}=b_{1} Y_{n} \oplus \ldots \oplus b_{n} Y_{n}$ be obtained with probability pe. Also

$$
\left|p_{e}-1 / 2\right|=\frac{2^{n-1}-N L(\Pi)}{2^{n}}
$$

For example if we have a SP network with 10 rounds and an $8 \times 8$ S-Box with $\mathrm{NL}(I I)=112$ like in AES, will be necessary at least $2^{62}$ known plain-text to obtain a bit of the key.

Differential Cryptanalysis In many of his papers Biham and Shamir show the witness of some ciphers against the differential cryptanalysis for this reasonis fundamental guaranties the protection against this kind of attack.

In [12] is defined the probability of the difference par $(\Delta \mathrm{X}, \Delta \mathrm{Y})$ in an S-Box to the probability that $\Delta Y$ occurs when one of the input values is selected randomly and the other is deduced from the relation $\Delta \mathrm{X}$. The probability of the most frequently par was denoted as $\mathrm{p} \delta$.

Many authors affirm that the minimization of $\mathrm{p} \delta$ brings resistance against differential cryptanalysis . O'Connor [13] shows that for a values of $n$, The probability of the difference par in an S-Box is expected less than the value $n / 2^{n-1}$. For example in an $8 \times 8 \mathrm{~S}$ - Box p $\delta$. $\leq 2^{-4}$.

Another frequently cause of high probability characteristic is the pour diffusion in the S-Box bits [13][12].

\section{S-BOX CHARACTERIZATION}

In this section we introduce some definitions that allow us characterize S-Boxes from the values obtained in the four criteria shown before.

Definition 6. Let II : GF( $\left.2^{\mathrm{n}}\right) \rightarrow \mathrm{GF}\left(2^{\mathrm{n}}\right)$ we called Parameter Set of II to the vector

$$
\Pi^{*}=\left(p_{e}, b n, p_{\delta}, \text { deg }\right)
$$

when the parameters $p e, b n, p \delta$, deg denote the static probability, branch number, characteristic probability and the algebraic degree of II respectively. 
International Journal of Soft Computing, Mathematics and Control (IJSCMC),Vol. 4, No. 3, August 2015

The definition shown before brings the necessary tools to introduce the next definition from whom will be characterize the $S$-Boxes.

Definition 7. Let $\Pi_{1}: G F\left(2^{n}\right) \rightarrow G F\left(2^{n}\right)$ and $\Pi_{2}: G F\left(2^{n}\right) \rightarrow G F\left(2^{n}\right)$. We say that $\Pi_{1}$ and $\Pi_{2}$ are parametric equivalents if:

$$
\Pi_{1 \mid}^{*}=\Pi_{2}^{*}
$$

Another important approach would be posses dynamic S-Boxes in the cipher. However in practice is very difficult find S-Boxes with an acceptable parameter set. With this porpoise is presented the next definition.

Definition 8. Let $\mathrm{II}_{1}: \mathrm{GF}(2 \mathrm{n}) \rightarrow \mathrm{GF}\left(2^{\mathrm{n}}\right)$ and $\mathrm{II}_{2}: \mathrm{GF}\left(2^{\mathrm{n}}\right) \rightarrow \mathrm{GF}\left(2^{\mathrm{n}}\right)$. We say that $\mathrm{II}_{1}$ y $\mathrm{II}_{2}$ are left affine equivalents if exists an affine function $A: \operatorname{GF}\left(2^{n}\right) \rightarrow \operatorname{GF}\left(2^{n}\right)$ so that:

$$
\Pi_{1}=A \circ \Pi_{2}
$$

Affine functions are very easy to construct, for this reason we could maybe think about obtain SBoxes with an acceptable parameter set from the composition of one with an acceptable parameter set selected a priory and an affine function. Take this into account is important to know the be haviour of this composition. For this reason we consider the next example.

if we have that $A^{-1}=\left(\begin{array}{lll}1 & 0 & 1 \\ 0 & 1 & 0 \\ 1 & 1 & 1\end{array}\right)$ then to $j_{1}=\left(\begin{array}{l}0 \\ 1 \\ 1\end{array}\right)$ and $j_{2}=\left(\begin{array}{l}1 \\ 1 \\ 0\end{array}\right)$ we obtain that:

$$
A^{-1} j_{1}=A^{-1} j_{2}=\left(\begin{array}{l}
1 \\
1 \\
0
\end{array}\right)
$$

The above example serves as a basis to demonstrate the following properties, which are an essential contribution to the development of this study.

Proposition 1. Let II1 : $\mathrm{GF}\left(2^{\mathrm{n}}\right) \rightarrow \mathrm{GF}\left(2^{\mathrm{n}}\right)$ and II2 $: \mathrm{GF}\left(2^{\mathrm{n}}\right) \rightarrow \mathrm{GF}\left(2^{\mathrm{n}}\right)$ S-Boxes left affine equivalent. Then we have that:

$$
p_{\delta}\left(\Pi_{1}\right) \geq p_{\delta}\left(\Pi_{2}\right)
$$


International Journal of Soft Computing, Mathematics and Control (IJSCMC),Vol. 4, No. 3, August 2015

Proof. Given that $\Pi_{1}$ and $\Pi_{2}$ are S-Boxes left affine equivalent then exists $A(x)=\hat{A}(x) \oplus C$ so that $\Pi_{1}=A \circ \Pi_{2}$.

$$
\begin{aligned}
p_{\delta}\left(\Pi_{1}\right) & =\max P\left(\Pi_{1}(x) \oplus \Pi_{1}(x \oplus i)=j\right) \\
& =X O R\left(\Pi_{1}\right) / 2^{n} \\
& =\max \left|x: \hat{A} \Pi_{2}(x) \oplus C \oplus \hat{A} \Pi_{2}(x \oplus i) \oplus C=j\right| / 2^{n} \\
& =\max \left|x: \Pi_{2}(x) \oplus \Pi_{2}(x \oplus i)=\hat{A}^{-1} j\right| / 2^{n}
\end{aligned}
$$

Now taking $j^{\prime}=\hat{A}^{-1} j$ we obtain that for each $(i, j)$ exists $\left(i, j^{\prime}\right)$ so that:

$$
\begin{aligned}
& \left|x: \Pi_{2}(x) \oplus \Pi_{2}(x \oplus i)=\hat{A}^{-1} j\right|=\left|x: \hat{A} \Pi_{2}(x) \oplus C \oplus \hat{A} \Pi_{2}(x \oplus i) \oplus C=j^{\prime}\right| \\
& \quad=\left|x: \Pi_{2}(x) \oplus \Pi_{2}(x \oplus i)=j^{\prime}\right|
\end{aligned}
$$

However could happend that for two differents par $(i, j)$ exist two equal par $\left(i, j^{\prime}\right)$ as we can see in the example before. For this reason we have that:

$$
\begin{aligned}
& \max X O R_{\Pi_{1}}(i, j) \geq \max X O R_{\Pi_{2}}\left(i, j^{\prime}\right) \\
& \geq p_{\delta}\left(\Pi_{2}\right)
\end{aligned}
$$

For static probability a similar result could be obtain:

Proposition 2. Let II1 $: \mathrm{GF}\left(2^{\mathrm{n}}\right) \rightarrow \mathrm{GF}\left(2^{\mathrm{n}}\right)$ and II2 $: \mathrm{GF}\left(2^{\mathrm{n}}\right) \rightarrow \mathrm{GF}\left(2^{\mathrm{n}}\right)$ S-Boxes left affine equivalents. Then we have that:

$$
p_{e}\left(\Pi_{1}\right) \geq p_{e}\left(\Pi_{2}\right) .
$$

Proof. Given that $\Pi_{1}$ and $\Pi_{2}$ are left affine equivalents then exist $A(x)=\hat{A}(x) \oplus$ $C$ so that $\Pi_{1}=A \circ \Pi_{2}$.

$$
\begin{aligned}
p_{e}\left(\Pi_{1}\right) & =1-\frac{N L\left(\Pi_{1}\right)}{2^{n}} \\
& =1-\frac{N L\left(\hat{A} \Pi_{2} \oplus C\right)}{2^{n}}
\end{aligned}
$$

In the other side we have that: 
International Journal of Soft Computing, Mathematics and Control (IJSCMC),Vol. 4, No. 3, August 2015

$$
\begin{aligned}
& =N L\left(\hat{A} \Pi_{2} \oplus C\right) \\
& =\min N L(z) \\
& =\min \left|x: a\left(\hat{A} \Pi_{2} \oplus C\right)=\omega x \oplus c\right| \\
& =\min \left|x: a \hat{A} \Pi_{2}=\omega x \oplus(c \oplus a C)\right|
\end{aligned}
$$

Now taking $a^{\prime}=a \hat{A}$ y $c^{\prime}=c \oplus a C$ we obtain that for any $z=(a, \omega, c)$ exist $z^{\prime}=\left(a^{\prime}, \omega, c^{\prime}\right)$ so that:

$$
\left|x: a\left(\hat{A} \Pi_{2} \oplus C\right)=\omega x \oplus c\right|=\left|x: a^{\prime}\left(\Pi_{1} \oplus C\right)=\omega x \oplus c^{\prime}\right|
$$

However could happend that for two differents tern $(a, \omega, c)$ exist two equal tern $\left(a^{\prime}, \omega, c^{\prime}\right)$. Then $N L\left(\Pi_{1}\right) \leq N L\left(\Pi_{2}\right)$ whereby

$$
p_{e}\left(\Pi_{2}\right) \leq p_{e}\left(\Pi_{1}\right)
$$

To see how is the behaviour of branch number of an S-BOX under left affine equivalents transformations we consider the next example:

Example 2. Let $\Pi_{1}: G F\left(2^{8}\right) \rightarrow G F\left(2^{8}\right)$ and $\Pi_{2}: G F\left(2^{8}\right) \rightarrow G F\left(2^{8}\right)$ left affine equivalents S-Boxes so that: $\Pi_{1}=A \circ \Pi$,

$$
A(x)=\left(\begin{array}{llllllll}
1 & 0 & 0 & 0 & 0 & 1 & 0 & 1 \\
0 & 1 & 0 & 0 & 0 & 0 & 0 & 0 \\
0 & 0 & 1 & 0 & 0 & 0 & 1 & 0 \\
0 & 0 & 0 & 1 & 0 & 1 & 0 & 0 \\
0 & 0 & 0 & 0 & 1 & 0 & 0 & 0 \\
0 & 0 & 0 & 0 & 0 & 1 & 0 & 0 \\
0 & 0 & 0 & 0 & 0 & 0 & 1 & 0 \\
0 & 0 & 0 & 0 & 0 & 0 & 0 & 1
\end{array}\right) \cdot x
$$

And 
International Journal of Soft Computing, Mathematics and Control (IJSCMC),Vol. 4, No. 3, August 2015

$\Pi=\{209,88,197,173,93,12,116,247,210,249,125,231,169,32,2,109,65$,

$8,79,158,60,204,105,224,233,196,96,166,175,149,213,16,18,31,97$,

$177,48,174,45,154,243,195,1,76,92,218,200,167,135,153,68,110$,

$121,70,141,251,203,144,220,201,14,55,108,245,13,75,21,72,238$,

$24,132,142,178,22,57,216,129,61,191,241,17,206,36,127,190,181$

, 112, $9,41,148,255,103,170,102,4,194,217,122,56,239,117,192$,

$185,147,33,137,90,104,43,165,160,53,146,140,171,67,54,119,214$

, 208, 62, 80, 23, 29, 69, 44, 159, 232, 221, 50, 234, 133, 237, 120, 11, 227,

$244,128,207,236,163,143,63,115,19,7,20,176,38,235,151,91,49,83$

, 183, 52, 64, 187, 161, 212, 35, 10, 86, 162, 100, 189, 40, 89, 71, 188, 46, 113,

$253,85,37,0,58,131,219,254,25,99,156,3,47,51,136,215,240,77,155$

, 168, 30, 59, 123, 134, 34, 225, 226, 179, 198, 164, 87, 182, 39, 107, 211, 124,

$193,229,66,94,172,73,28,98,152,246,106,252,130,114,145,27,95,111$

, 5, 230, 184, 42, 199, 74, 84, 157, 139, 250, 81, 223, 248, 118, 138, 126, 180 ,

$82,242,228,78,205,186,6,101,15,26,150,202,222$,

However is easy to see that: $\left(b n\left(\Pi_{1}\right)=2\right) \neq\left(b n\left(\Pi_{2}\right)=3\right)$ Attending to the results parametric equivalents transformations are not possible to classify by the left composition with affine transformations.

\section{PRESENTATION OF THE NEW ALGORITHM}

As we show before S-Boxes with good diffusion in the output bits posses a better resistance against differential cryptanalysis. The branch number is an S-Box design criterion that results as a diffusion measure. Unfortunately experimental results show that S-Boxes with non-trivial diffusion orders cant be determinate by randomly search $(\mathrm{b} n>2)$.

Table 1. Probability of randomly selection of S-Boxes with $b n \geq 3$

\begin{tabular}{cc}
\hline n & Valor estimado \\
\hline 3 & $1.2 \times 10^{-3}$ \\
4 & $4.9 \times 10^{-5}$ \\
5 & $7.7 \times 10^{-7}$ \\
6 & $14.5 \times 10^{-9}$ \\
7 & $9.3 \times 10^{-12}$ \\
8 & $6.7 \times 10^{-15}$
\end{tabular}


International Journal of Soft Computing, Mathematics and Control (IJSCMC),Vol. 4, No. 3, August 2015 A new algorithm for the construction of S-Boxes with $b n=3$ is presented below.

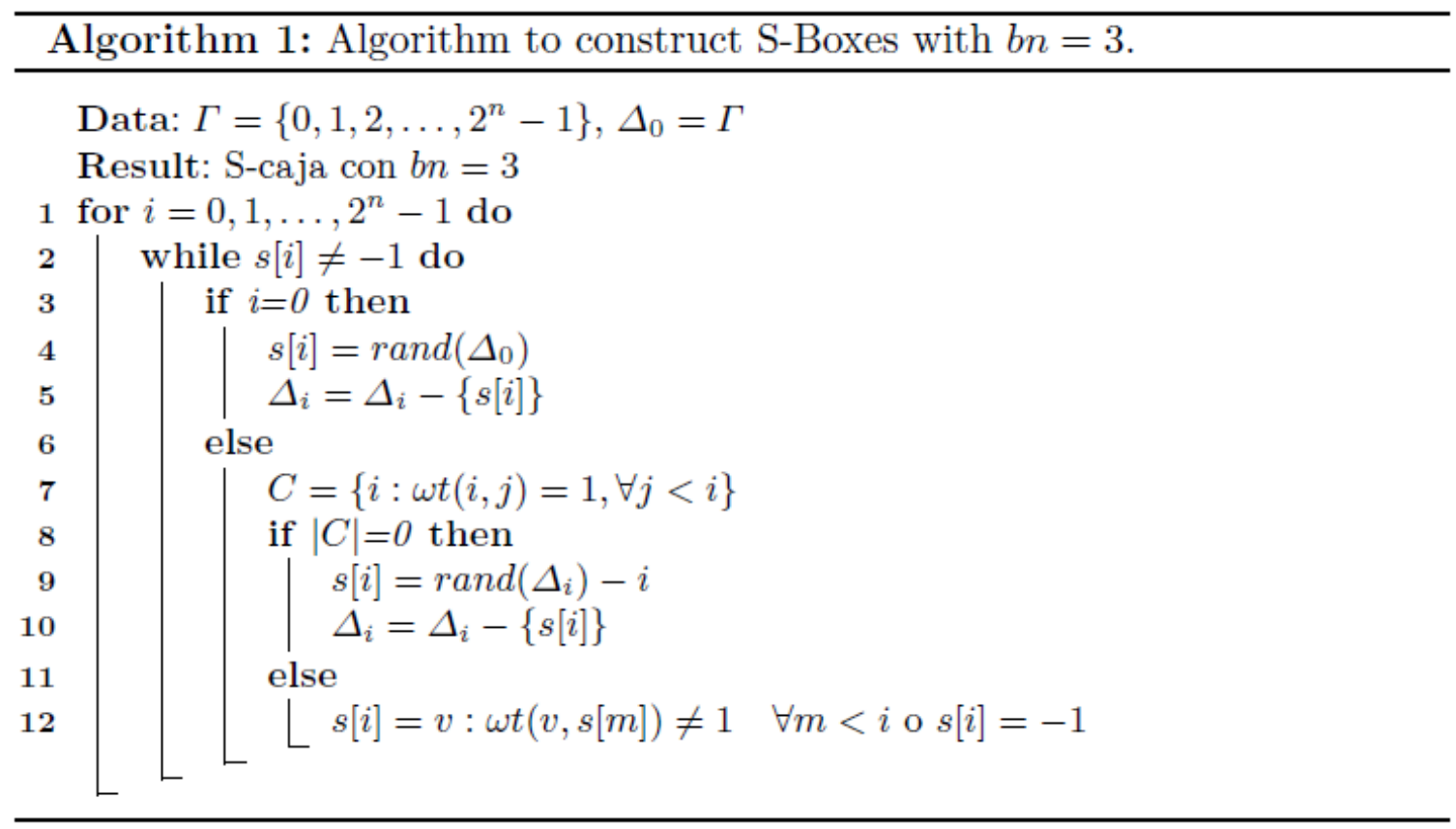

For a better understanding of the algorithm we consider the next sets: $L_{i}=\{(a, b): w t(a \oplus b)=i\}$

$\mathrm{B}(i)$ is the set formed by the images of the elements less equal than $\mathrm{i}$.

Then the construction is performed as follows:

A random value different to 0 is assigned to the S-box for input 0 .

$$
f(0)=\operatorname{rand}(255) \neq 0
$$

Then if $(i, k) \in L_{2}, \forall k<i$ is assigned to $f(i)$ a random value different to $i$ not belonging to the set $f(k): k<i$.

$$
f(i)=\operatorname{rand}(255) \notin B(i-1) \neq i
$$

In the other way if ${ }^{\mathrm{a}} K<i$ so that $(i, k) € \mathrm{~L}_{1}$.

$$
f(i)=x_{i} \neq i:\left(x_{i}, f(k)\right) \in L_{2}
$$


International Journal of Soft Computing, Mathematics and Control (IJSCMC),Vol. 4, No. 3, August 2015

\subsection{Comparative results}

In [12] Tavares propose an algorithm for the construction of S-Boxes with $b n \geq 3$, however this algorithm present some inconvenient. The $8 \times 8$ constructed S-Boxes could posses a high quantity of fixed points. Also the nonlinearity values for this constructions are always less equal than 96 as we can see in the table 3 .

Table 2. Nonlinearity of $8 \times 8$ S-Boxes in the new construction.

\begin{tabular}{ccccccc}
\hline Parameters & \multicolumn{6}{c}{ bn Min $N L$ Max $N L \% N L=96 \% N L=98 \% N L=100$} \\
\hline Tavares Algorithm & 3 & 86 & 96 & 26 & 0 & 0 \\
\hline New Algorithm & 3 & 92 & 100 & 36 & 14 & 0.5 \\
\hline
\end{tabular}

We generate a set of $50 \mathrm{~S}$-Boxes in which we find his nonlinearity values and the results are showed in the table 3 .

Is easy to see that if we compare the result of table 3 the output S-boxes of the new algorithm posses nonlinearity values higher than the S-boxes obtained by the Tavares algorithm.

Table 3. Comparision between the new algorithm and the Tavares proposal.

\begin{tabular}{ccc}
\hline Parameters & Tavares algorithm New & Algorithm \\
\hline Max nonlinearity & 96 & 100 \\
\hline Fixed Points & yes & no \\
\hline Kind of algorithm & search & constructive \\
\hline
\end{tabular}

\section{CONCLUSIONS}

In this paper is presented an algorithm to generate S-boxes with high diffusion. Also was shown that these outputs could have acceptables values of nonlinearity and do not have fixed point wich allows that these S-boxes could be used as part of the cipher in a secure way.

As a theoretical result was proved that the Parameter Set of an S-box is not invariant under left affine transformations. Take this into account is not possible obtain S-boxes with a good Parameter Set by the left composition with an affine function and a good S-box.

Finally was shown that the outputs of the new algorithm have better results than the outputs of the Tavares algorithm. 
International Journal of Soft Computing, Mathematics and Control (IJSCMC),Vol. 4, No. 3, August 2015

\section{REFERENCES}

[1] Daemen J. and Rijmen V. The Rijndael block cipher. AES Proposal. http/www.daimi.ai.dk/iran/rijndael.pdf. 1999.

[2] Mister.S. \& Adams.C. Practical S-Box Design. Nortel, P.O. Box 3511, Station C, Canada. 1990.

[3] Webster.A.F. \& Tavares.S.E. On the design of S-Boxes. Department of Electrical Engineering Queen's University. 1989.

[4] Heys.H. \& Tavares.S.E. Avalanche Characteristics of Substitution-Permutation Encryption Networks. IEEE. 1991.

[5] Vergili.I \& Yucel.M.D. Statistics on satisfaction of Security Criteria for RandomlyChosen S-Boxes. Middle East Technical University. 2000.

[6] Daemen.J. \& Rijmen.V. The Design of Rijndael. Springer. 2002.

[7] Przemyslaw.R. \& Mroczkowski.P. How to create goog s-boxes. 1st International Conference for Young Researchers. 2006.

[8] Nyberg.K.Dierentially uniform mappings for cryptography. Institute of Computer Technology, Vienna Technical University. 1994.

[9] Shamir.A.\&Biham.E.Dierential Cryptanalysis of DES-like Cryptosystems. Journal of Cryptology. Vol.4, No 1. 1991.

[10] Matsui.M. Linear cryptanalysis method for DES cipher. Advances in Cryptology: Proceedings of EUROCRYPT 93, Spriger-Verlag, Berlin. 1994.

[11] Perez, Claudia and Gainzo, Felipe. Algunos criterios para determinar la seguridad en las S-cajas. Memorias del evento Compumat 2013.

[12] Heys. H.M \& Tavares. S. E. Substitution-Permutation Networks Resistant to Differential and Linear Cryptanalysis. Department of Electrical and Computer Engineering, Queens University, Kingston, Ontario, Canada K7L 3N6. 1994.

[13] OConnor. L. J. On the distribution of characteristics in bijective mappings. Advances in Cryptology: Proceedings of EUROCRYPT 93, Springer-Verlag, Berlin, pages 360370. 1994.

[14] Youssef. A.M., Mister. S. \& Tavares. S.E. On the Design of Linear Transformations for Substitution Permutation Encryption Networks. Department Of Electrical and Computer Engineering Queens University, Kingston, Ontario, Canada. 1998. 